\title{
Karyotype variation in lesser short-nosed fruit bat Cynopterus brachyotis (Müller 1838) from Special Region Yogyakarta, Indonesia
}

\author{
HUSNI MUBAROK ${ }^{1}$, NIKEN SATUTI NUR HANDAYANI ${ }^{1}$, IBNU MARYANTO ${ }^{2}$, TUTY ARISURYANTI ${ }^{1, \bullet}$ \\ ${ }^{1}$ Department of Tropical Biology, Faculty of Biology, Universitas Gadjah Mada, Jl. Teknika Selatan, Sekip Utara, Sleman 55281, Yogyakarta, Indonesia. \\ Tel.: +62-274-580839, Fax.: +62-274-6492354, `email: tuty-arisuryanti@ugm.ac.id \\ ${ }^{2}$ Museum Zoologicum Bogoriense, Widyasatwaloka Building, Research Center for Biology, Indonesian Institute of Sciences. Jl. Raya Jakarta-Bogor Km. \\ 46, Cibinong, Bogor 16911, West Java, Indonesia.
}

Manuscript received: 18 March 2021. Revision accepted: 15 April 2021.

\begin{abstract}
Mubarok H, Handayani NSN, Maryanto I, Arisuryanti T. 2021. Karyotype variation in lesser short-nosed fruit bat Cynopterus brachyotis (Müller 1838) from Special Region Yogyakarta, Indonesia. Biodiversitas 22: 2560-2568. Lesser short-nosed fruit bat Cynopterus brachyotis is a widespread fruit bat in different habitats. This species is suspected to have chromosome morphology variation, since karyotype data in Indochinese Peninsula, Borneo, West Java, and the Philippines has been revealed. Karyotype data can provide basic information on taxonomic relationships, evolutionary origins, and genetic aberrations. This study aimed to analyze karyotype variation in C. brachyotis from different habitats in a special region of Yogyakarta, Indonesia. Total 14 sample bats were mist-nets trapped, including six individuals from an urban area, three individuals from natural forest, and five individuals from mangrove forest. Chromosome preparations were conducted using the splash method with bone marrow, including treatment of colchicine $0.005 \%, 0.075 \mathrm{M} \mathrm{KCl}$ solution, Carnoy solution, and Giemsa staining. Chromosome was analyzed and classified using ImageJ Levan Plugin software. The result showed C. brachyotis from different habitats in this study indicated karyotype variation and have different karyotype from West Java samples. Males C. brachyotis from mangrove forest have a karyotype formula of $2 \mathrm{n}=34$, $\mathrm{FN}=61,16 \mathrm{~m}+8 \mathrm{sm}+2 \mathrm{st}+6 \mathrm{t}, \mathrm{X}=\mathrm{st}$, and $\mathrm{Y}=\mathrm{t}$, while female $C$. brachyotis from the urban area have karyotype formula of $2 \mathrm{n}=34, \mathrm{FN}=62$, $16 \mathrm{~m}+4 \mathrm{sm}+6 \mathrm{st}+6 \mathrm{st}$, and $\mathrm{X}=\mathrm{st}$.
\end{abstract}

Keywords: Bats, Chromosome, Cynopterus brachyotis, Karyotype, Variation.

\section{INTRODUCTION}

There are seven species of genus Cynopterus in the world, namely $C$. brachyotis, $C$. titthaecheilus, $C$. sphinx, C. luzoniensis, C. nusatenggara, $C$. horsfieldii, and $C$. minutus. All of these species have been distributed in Indonesia (Burgin et al. 2020; Simmons and Cirranello 2020). Moreover, five species of this genus (except $C$. luzoniensis and $C$. nusatenggara) can be found in the special region Yogyakarta (Suripto et al. 2001; Suripto et al. 2006; Mubarok 2019, unpublished data).

C. brachyotis or known as lesser short-nosed fruit bat belongs to the family Pteropodidae which inhabit different types of habitat (euritropics) from the urban, forest, and mangrove area. This species disperses seed and pollinates more than ten different plants in the ecosystem, such as Acanthaceae, Anacardiaceae, Euphorbiaceae, Fabaceae, Loranthaceae, Myrtaceae, Rubiaceae, Sapindaceae, and more (Maryanto 1993; Simmons 2005; Maryati et al. 2008). C. brachyotis in Indonesia only distributed to Sumatra, Java, Bali, Sulawesi, and Kalimantan (Kitchener and Maharadatunkamsi 1991; Suyanto et al. 2002; Maryanto and Higashi 2011). This species in Thailand, Malaysia, Sumatra, Sulawesi, Borneo, Philippines, and other islands have reported to having morphological variations, especially in forearm length, tail length, tibia length, and body weight (Andersen 1912; Abdullah 2003; Francis et al. 2010). There are two different noted forms of
C. brachyotis based on morphological and genetic that occupy various habitats in Indochinese Peninsula and Borneo, known as $C$. brachyotis Sunda and C. brachyotis Forest (Abdullah 2003; Campbell et al. 2004).

Morphological variations occurred in C. brachyotis, but several karyotype studies showed this species from Malaysia, the Philippines and Java have a uniformly number of diploid chromosomes (2n). C. brachyotis typically have $2 \mathrm{n}=34$, subtelocentric $X$ chromosome, and acrocentric Y chromosome (Yong et al. 1973; Ando et al. 1980; Harada and Kobayashi 1980). However, the $X$ chromosome of Philippines bats have been classified as submetacentric and sex chromosome of $C$. brachyotis from Malaysia were not determined (Harada and Kobayashi 1980; Rickart et al. 1999). It is indicated that karyotype variations may also occur in C. brachyotis, especially in chromosome shape.

The first karyotype study of Indonesian fruit bats has conducted by Ando et al. (1980), including C. brachyotis species. Four male and three female C. brachyotis were collected from West Java. According to this study, metacentric and submetacentric chromosomes were not sharply distinguished, Therefore, the chromosome shape classification of this species in Indonesia still not clear. Besides, no recent study to reveal karyotype study of this species particularly from another locality for decades.

A recent phylogenetic study of the genus Cynopterus in Indonesia has been revealed that $C$. brachyotis, $C$. minutus, 
and $C$. luzoniensis were not represented as different cohesive clusters and they were not corresponding with recognized morphological characters (Zein and Fitriana 2015). The morphological, chromosomal study and genetic studies can be carried out to clarify taxonomic relationships among fruit bats species. Karyotype analysis may give information about the taxonomic relationships between species, evolutionary origin, and also genetic aberrations (Young et al. 2012). Since C. brachyotis is suspected to have different karyotypes among individuals in species, karyotype variation may indicate subgenomic and ecotypic differentiation in a population of the organism (Young et al. 2012). Therefore, this study aimed to analyze karyotype variation in C.brachyotis collected from different habitats in the special region of Yogyakarta, Indonesia.

\section{MATERIALS AND METHODS}

\section{Capture site and animal ethics}

Lesser short-nosed bats were captured from November to December 2019 at three locations with different habitat types comprising of (i) urban area Bulaksumur, Sleman (745'57.2”S, 110²2’32.8’E), (ii) Tlogo Nirmolo Natural Forest of Merapi National Park, Sleman (7³5'24.2”S, $\left.110^{\circ} 25^{\prime} 35.7^{\prime} \mathrm{E}\right)$, and Wanatirta Mangrove Forest, Kulon Progo (7'53'32.9'S, 110 01'10.1'E) in Yogyakarta Province, Indonesia (Figure 1).
A total of 14 individuals C. brachyotis were collected and processed for karyotype analysis, including (i) one adult male, four adult females, and one sub-adult female from an urban area, (ii) two adult males and one juvenile female from natural forest, also (iii) two adult males, one juvenile male, one adult female, and one juvenile female from mangrove area (Table 1). The sample of lesser shortnosed fruit bats in each capture site is presented in Figure 2. Animal handling and treatments in this study were done under the Research Ethics Committee of the Faculty of Veterinary Medicine, Universitas Gadjah Mada, Indonesia permit number 0108/ EC-FKH/ Ex./ 2019.

\section{Animal sampling and identification}

Bats were captured at dusk using mist nets ( $9 \mathrm{~m}$ wide $\mathrm{X}$ $2.5 \mathrm{~m}$ deep) that were placed close to Muntingia calabura and Ficus sp. at 3-4 m above ground. Each bat is released carefully from the net then put in a canvas bag for further analysis. Species identification was conducted based on Suyanto (2001) dan Huang et al. (2016). Physical examinations including sex (male and female), age class (adult, sub-adult, juvenile, and infant), and also morphological measurement (head and body length, tail to ventral length, forearm length, tibia length, ear height, first digit with claw length, hindfoot and body weight) were also conducted.

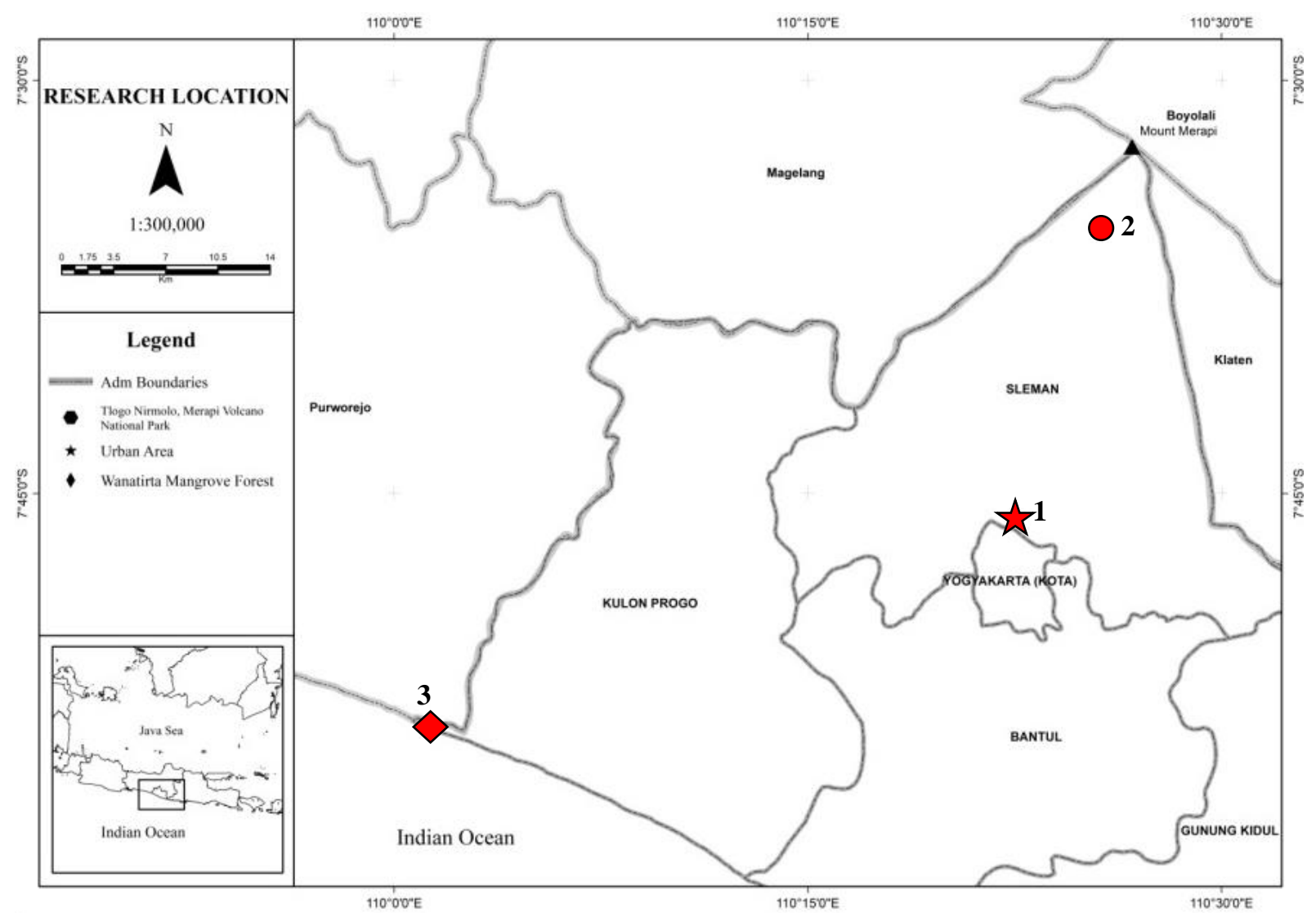

Figure 1. Research location of this study in Yogyakarta Province, Indonesia. 1. Urban area of Bulaksumur, Sleman, 2. Tlogo Nirmolo Natural Forest of Merapi National Park, Sleman, 3. Wanatirta Mangrove Forest, Kulon Progo 
A
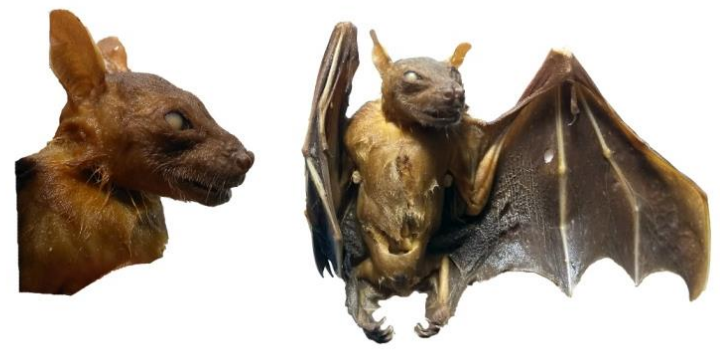

B
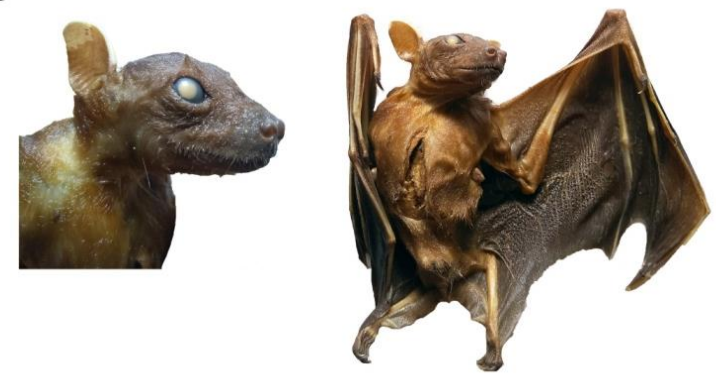

C
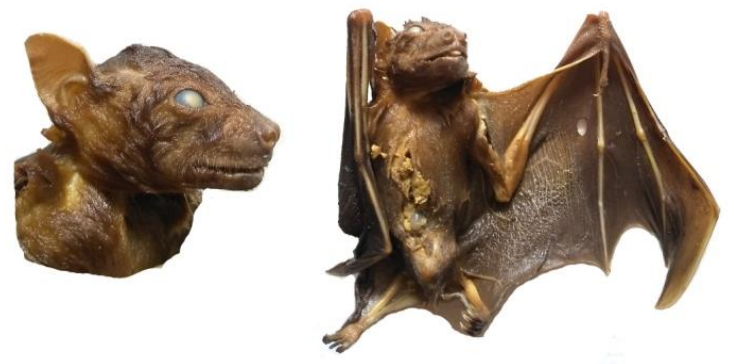

Figure 2. Sample of lesser short-nosed fruit bats $C$. brachyotis in each capture site. A. Urban area Bulaksumur, Sleman, B. Tlogo Nirmolo natural forest Merapi volcano national park, Sleman, C. Wanatirta mangrove forest, Kulon Progo.

\section{Karyotype analysis}

Chromosome preparation was conducted in fieldwork using the splash method from Baker et al. (1982) with modification. Each bat was injected with $0.005 \%$ mitotic inhibitors Colchicine (Sigma C9754) intraperitoneally at a dose of $0.1 \mathrm{~mL} / 10 \mathrm{~g}$ body weight for two hours. Bats were anesthetized with Ketamine-Xylazine at a dose of $50 \mathrm{mg} /$ $\mathrm{kg}$ body weight and then sacrificed using cervical dislocation. Metaphase stages were collected from the bone marrow humerus. Bone marrow was treated in $0.075 \mathrm{M}$ $\mathrm{KCl}$ solution for at least 45 minutes then centrifuged at $1200 \mathrm{rpm}$ for 10 minutes. Hereinafter, cell pellet was fixed using Carnoy solution (3 methanol : 1 acetate acid glacial) for 15 minutes and recentrifuged. Resuspension cell pellet using new Carnoy solution then splashed on slides and airdried. Staining slides were performed using $4 \%$ Giemsa for 30 minutes.

The best metaphase plates were observed on a microscope within 1000 magnification. Diploid number $(2 \mathrm{n})$, fundamental number $(\mathrm{FN})$, and fundamental number autosome ( $\mathrm{FNa}$ ) were calculated manually. Short and long arm length, total length, arm ratio (AR), and chromosome shape were classified automatically into metacentric (M; $\mathrm{AR}=1.0-<1.7$ ), submetacentric ( $\mathrm{SM} ; \mathrm{AR}=1.7 \quad-<3.0$ ), subtelocentric (ST; AR=3.0 - 7.0 ) and telocentric (T; $\mathrm{AR}=\infty$ ) using ImageJ software with Levan Plugin (Levan et al. 1964; Abramoff et al. 2004; Sakamoto and Zacaro 2009), free downloaded at http://rsbweb.nih.gov/ij/download.html. The centromeric index and relative length of the chromosome were calculated using Microsoft Excel software. The chromosome karyotypes were arranged according to their shape and length. Since there is no proper numbering of chromosomes in fruit bats, we gave the number based on chromosome morphology, from metacentric to telocentric.

Table 1. Number of $C$. brachyotis sample studied and its physical examination that collected from sampling sites

\begin{tabular}{|c|c|c|c|c|c|c|c|c|c|c|c|c|}
\hline \multirow{2}{*}{$\begin{array}{c}\text { Sampling } \\
\text { code }\end{array}$} & \multirow{2}{*}{ Habitat } & \multirow{2}{*}{$\begin{array}{l}\text { Capture } \\
\text { Site }\end{array}$} & \multirow{2}{*}{ Sex } & \multirow{2}{*}{ Age } & \multicolumn{8}{|c|}{ External morphology measurement (mm) } \\
\hline & & & & & FA & FDL & EH & TVL & TL & HF & HBL & BW (g) \\
\hline CB1 & Urban & Bulaksumur & Female & Sub-adult & 61.6 & 24.1 & 17.9 & 11.4 & 21.8 & 13.1 & 87.6 & 30.6 \\
\hline CB2 & area & Sleman & Female & Adult & 65.6 & 24.8 & 13.2 & 11.5 & 21.8 & 14.2 & 81.1 & 35.2 \\
\hline CB3 & & & Male & Juvenile & 60.5 & 25.1 & 11.5 & 10.7 & 21.9 & 13.7 & 72.8 & 25.6 \\
\hline CB4 & & & Female & Adult & 64.7 & 27.5 & 15.0 & 12.0 & 25.4 & 14.9 & 85.1 & 36.8 \\
\hline CB5 & & & Female & Adult & 64.8 & 26.9 & 16.1 & 11.1 & 26.6 & 16.2 & 74.4 & 38.6 \\
\hline CB6 & & & Female & Adult & 65.6 & 18.8 & 12.2 & 11.4 & 24.3 & 16.7 & 74.1 & 39.0 \\
\hline CB7 & Natural & Tlogo & Male & Adult & 68.1 & 28.4 & 12.3 & 13.0 & 26.3 & 14.1 & 95.0 & 51.1 \\
\hline CB8 & forest & Nirmolo & Male & Adult & 64.3 & 26.0 & 14.0 & 11.6 & 25.6 & 16.3 & 88.9 & 44.8 \\
\hline CB9 & & & Female & Juvenile & 60.5 & 28.5 & 13.9 & 10.9 & 20.4 & 19.0 & 81.2 & 35.4 \\
\hline CB10 & Mangrove & Wanatirta & Male & Juvenile & 60.8 & 26.6 & 15.6 & 12.2 & 21.0 & 13.9 & 67.3 & 30.2 \\
\hline CB11 & forest & mangrove, & Male & Adult & 63.9 & 25.0 & 15.5 & 10.6 & 24.0 & 13.0 & 84.4 & 43.7 \\
\hline CB12 & & Kulon & Female & Adult & 68.0 & 26.5 & 17.0 & 11.5 & 26.2 & 12.6 & 85.0 & 43.5 \\
\hline CB13 & & Progo & Female & Juvenile & 60.9 & 23.1 & 15.8 & 11.5 & 21.5 & 12.2 & 84.3 & 29.6 \\
\hline CB14 & & & Male & Adult & 66.5 & 25.0 & 16.5 & 11.8 & 24.9 & 16.3 & 84.8 & 36.7 \\
\hline
\end{tabular}

Note: FA: Forearm length, FDL: First digit length (with claw), EH: Ear height, TVL: Tail to ventral length, TL: Tibia length, HF: Hindfoot length, HBL: Head and body length, and BW: Bodyweight 


\section{RESULTS AND DISCUSSION}

\section{Karyotype of $C$. brachyotis}

Only three individuals from two localities showed the best karyotype. Unfortunately, one individual bat from the mangrove forest showed three best metaphase plates (adult) and the other showed only one (juvenile). One best metaphase plate can be observed in an individual bat from an urban area. Moreover, all karyotypes of $C$. brachyotis from the natural forest can not be analyzed. Nevertheless, both $C$. brachyotis that collected from mangrove forest and urban area is indicated karyotypes variation. Karyotypic trends for each chromosome are detailed below.

\section{C. brachyotis from mangrove forest}

This individual has diploid number $(2 n)=34, F N=61$, $\mathrm{FNa}=58$. Autosomes comprised eight pairs of large to small metacentric, four pairs of medium submetacentric, one pair of small subtelocentric, and three pairs of minute telocentric for adult bat (Figure 2) and juvenile bat (Figure 3). Marker chromosomes are classified as submetacentric and having secondary constrictions close to centromeres. The $\mathrm{X}$ chromosome is medium subtelocentric and the $\mathrm{Y}$ chromosome is telocentric. Therefore, the karyotype formula of this species from the mangrove forest was $2 \mathrm{n}=34 ; 16 \mathrm{~m}+8 \mathrm{sm}+2 \mathrm{st}+6 \mathrm{t} ; \mathrm{X}=\mathrm{st}, \mathrm{Y}=\mathrm{t}$.

The highest relative length of chromosome in adult male was metacentric which $6.78 \pm 0.59$ (Table 2), while in juvenile male was 5.50 (Table 3 ). The relative length of marker chromosomes in juvenile males took the fourth position of largest relative length in the karyotype. Meanwhile, in adult male was fifth. X chromosome in adult males has a relative length near to submetacentric size (chromosome-11A).

\section{C. brachyotis from urban area}

The karyotype of $C$. brachyotis from an urban area has a diploid number, $2 \mathrm{n}=34, \mathrm{FN}=62, \mathrm{FNa}=58$. Autosomes consisted of eight pairs metacentric, two pairs submetacentric, three pairs subtelocentric and telocentric, respectively (Figure 4). A pair of submetacentric chromosomes classified as a marker chromosome and their relative length position as well as a marker chromosome in a juvenile male bat from the mangrove area but smaller, about 3.66 (Table 4). The $X$ chromosomes of this species are medium subtelocentric. Hence, $C$. brachyotis from an urban area has karyotype formula $2 n=34,16 m+4 s m$ $+6 \mathrm{st}+6 \mathrm{st}, \mathrm{X}=\mathrm{st}$.

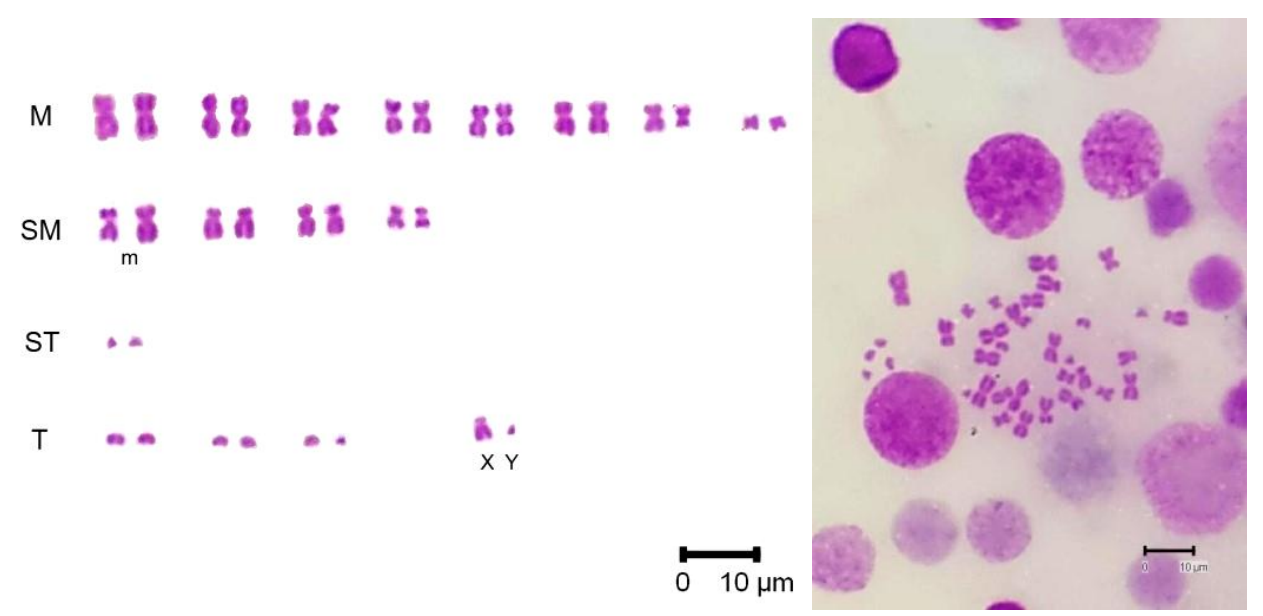

Figure 3. Karyotype of Cynopterus brachyotis adult male (CB14) from the mangrove forest, m: marker chromosomes (scale bar $10 \mu \mathrm{m})$

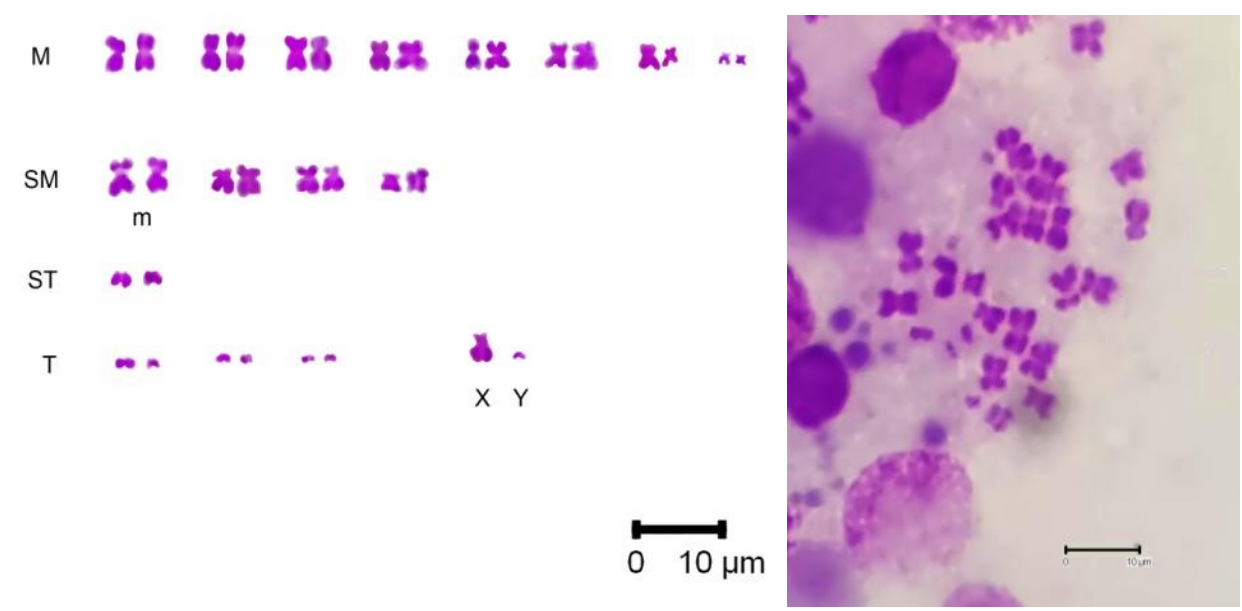

Figure 4. Karyotype of Cynopterus brachyotis juvenile male (CB10) from the mangrove forest, m: marker chromosomes (scale bar $10 \mu \mathrm{m})$ 

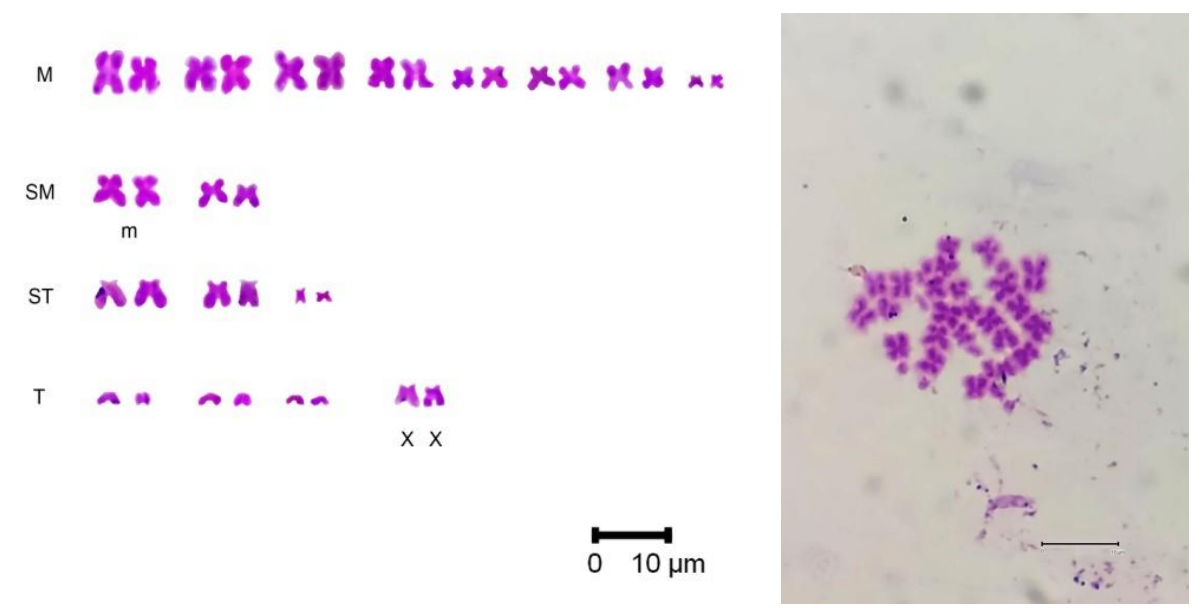

Figure 5. Karyotype of Cynopterus brachyotis adult female (CB6) from the urban area, m: marker chromosomes (scale bar $10 \mu \mathrm{m})$.

Table 2. Chromosome measurement and morphology of adult male $C$. brachyotis collected from the mangrove forest

\begin{tabular}{|c|c|c|c|c|c|c|c|}
\hline Chromosome & $\mathbf{p}$ & $\mathbf{q}$ & pq & $\mathbf{A R}$ & CI & RL\% & $\begin{array}{c}\text { Chromosome } \\
\text { shape }\end{array}$ \\
\hline $1 \mathrm{~A}$ & $3.09 \pm 0.37$ & $3.60 \pm 0.22$ & $6.69 \pm 0.58$ & $1.17 \pm 0.07$ & $0.46 \pm 0.02$ & $6.78 \pm 0.59$ & $\vec{M}$ \\
\hline 1B & $2.91 \pm 0.35$ & $3.31 \pm 0.32$ & $6.22 \pm 0.67$ & $1.14 \pm 0.03$ & $0.47 \pm 0.01$ & $6.30 \pm 0.68$ & M \\
\hline $2 \mathrm{~A}$ & $2.58 \pm 0.29$ & $3.09 \pm 0.10$ & $5.67 \pm 0.19$ & $1.22 \pm 0.19$ & $0.45 \pm 0.04$ & $5.74 \pm 0.19$ & M \\
\hline $2 \mathrm{~B}$ & $2.46 \pm 0.09$ & $2.75 \pm 0.10$ & $5.21 \pm 0.13$ & $1.12 \pm 0.06$ & $0.47 \pm 0.01$ & $5.28 \pm 0.13$ & M \\
\hline $3 \mathrm{~A}$ & $2.26 \pm 0.22$ & $2.66 \pm 0.25$ & $4.92 \pm 0.35$ & $1.19 \pm 0.17$ & $0.46 \pm 0.03$ & $4.98 \pm 0.35$ & M \\
\hline 3B & $2.06 \pm 0.15$ & $2.81 \pm 0.22$ & $4.87 \pm 0.37$ & $1.36 \pm 0.01$ & $0.42 \pm 0.00$ & $4.93 \pm 0.37$ & M \\
\hline $4 \mathrm{~A}$ & $2.00 \pm 0.17$ & $2.43 \pm 0.17$ & $4.43 \pm 0.16$ & $1.23 \pm 0.18$ & $0.45 \pm 0.03$ & $4.48 \pm 0.16$ & M \\
\hline $4 \mathrm{~B}$ & $1.90 \pm 0.15$ & $2.25 \pm 0.03$ & $4.15 \pm 0.16$ & $1.19 \pm 0.08$ & $0.46 \pm 0.02$ & $4.20 \pm 0.17$ & M \\
\hline $5 \mathrm{~A}$ & $1.72 \pm 0.11$ & $2.10 \pm 0.16$ & $3.82 \pm 0.26$ & $1.22 \pm 0.06$ & $0.45 \pm 0.01$ & $3.86 \pm 0.26$ & M \\
\hline $5 \mathrm{~B}$ & $1.59 \pm 0.24$ & $2.14 \pm 0.06$ & $3.73 \pm 0.29$ & $1.37 \pm 0.16$ & $0.42 \pm 0.03$ & $3.78 \pm 0.29$ & M \\
\hline $6 \mathrm{~A}$ & $1.56 \pm 0.11$ & $1.98 \pm 0.17$ & $3.54 \pm 0.19$ & $1.28 \pm 0.16$ & $0.44 \pm 0.03$ & $3.58 \pm 0.19$ & M \\
\hline $6 \mathrm{~B}$ & $1.46 \pm 0.14$ & $1.94 \pm 0.17$ & $3.40 \pm 0.24$ & $1.34 \pm 0.15$ & $0.43 \pm 0.03$ & $3.44 \pm 0.24$ & M \\
\hline $7 \mathrm{~A}$ & $1.28 \pm 0.11$ & $1.52 \pm 0.13$ & $2.80 \pm 0.15$ & $1.20 \pm 0.17$ & $0.46 \pm 0.04$ & $2.84 \pm 0.15$ & M \\
\hline $7 \mathrm{~B}$ & $1.16 \pm 0.09$ & $1.33 \pm 0.12$ & $2.49 \pm 0.20$ & $1.16 \pm 0.05$ & $0.46 \pm 0.01$ & $2.52 \pm 0.20$ & M \\
\hline $8 \mathrm{~A}$ & $0.89 \pm 0.19$ & $0.98 \pm 0.22$ & $1.87 \pm 0.41$ & $1.09 \pm 0.02$ & $0.48 \pm 0.01$ & $1.89 \pm 0.42$ & M \\
\hline $8 \mathrm{~B}$ & $0.82 \pm 0.15$ & $0.96 \pm 0.29$ & $1.79 \pm 0.44$ & $1.15 \pm 0.13$ & $0.47 \pm 0.03$ & $1.81 \pm 0.44$ & M \\
\hline $9 A^{*}$ & $1.25 \pm 0.07$ & $2.64 \pm 0.21$ & $3.89 \pm 0.27$ & $2.12 \pm 0.09$ & $0.32 \pm 0.01$ & $3.94 \pm 0.28$ & SM \\
\hline $9 \mathrm{~B}^{*}$ & $1.10 \pm 0.08$ & $2.25 \pm 0.23$ & $3.35 \pm 0.20$ & $2.07 \pm 0.33$ & $0.33 \pm 0.03$ & $3.40 \pm 0.20$ & SM \\
\hline $10 \mathrm{~A}$ & $1.14 \pm 0.16$ & $2.34 \pm 0.25$ & $3.48 \pm 0.40$ & $2.07 \pm 0.06$ & $0.33 \pm 0.01$ & $3.52 \pm 0.41$ & SM \\
\hline $10 \mathrm{~B}$ & $1.05 \pm 0.06$ & $2.27 \pm 0.32$ & $3.33 \pm 0.34$ & $2.16 \pm 0.30$ & $0.32 \pm 0.03$ & $3.37 \pm 0.34$ & SM \\
\hline $11 \mathrm{~A}$ & $0.79 \pm 0.11$ & $1.81 \pm 0.16$ & $2.59 \pm 0.14$ & $2.35 \pm 0.42$ & $0.30 \pm 0.04$ & $2.62 \pm 0.15$ & SM \\
\hline $11 \mathrm{~B}$ & $0.74 \pm 0.02$ & $1.61 \pm 0.09$ & $2.35 \pm 0.12$ & $2.17 \pm 0.07$ & $0.32 \pm 0.01$ & $2.38 \pm 0.12$ & SM \\
\hline $12 \mathrm{~A}$ & $0.64 \pm 0.08$ & $1.36 \pm 0.27$ & $2.01 \pm 0.32$ & $2.11 \pm 0.34$ & $0.32 \pm 0.03$ & $2.03 \pm 0.32$ & SM \\
\hline $12 \mathrm{~B}$ & $0.63 \pm 0.12$ & $1.25 \pm 0.27$ & $1.88 \pm 0.38$ & $1.96 \pm 0.07$ & $0.34 \pm 0.01$ & $1.91 \pm 0.39$ & SM \\
\hline $13 \mathrm{~A}$ & $0.26 \pm 0.05$ & $1.20 \pm 0.20$ & $1.46 \pm 0.24$ & $4.62 \pm 0.36$ & $0.18 \pm 0.01$ & $1.48 \pm 0.25$ & $\mathrm{ST}$ \\
\hline $13 \mathrm{~B}$ & $0.26 \pm 0.05$ & $1.00 \pm 0.21$ & $1.26 \pm 0.25$ & $3.79 \pm 0.09$ & $0.21 \pm 0.00$ & $1.28 \pm 0.26$ & ST \\
\hline $14 \mathrm{~A}$ & $0.00 \pm 0.00$ & $1.14 \pm 0.22$ & $1.14 \pm 0.22$ & $\infty$ & $0.00 \pm 0.00$ & $1.16 \pm 0.22$ & $\mathrm{~T}$ \\
\hline $14 B$ & $0.00 \pm 0.00$ & $1.06 \pm 0.20$ & $1.06 \pm 0.20$ & $\infty$ & $0.00 \pm 0.00$ & $1.08 \pm 0.20$ & $\mathrm{~T}$ \\
\hline $15 \mathrm{~A}$ & $0.00 \pm 0.00$ & $1.00 \pm 0.25$ & $1.00 \pm 0.25$ & $\infty$ & $0.00 \pm 0.00$ & $1.01 \pm 0.25$ & $\mathrm{~T}$ \\
\hline $15 B$ & $0.00 \pm 0.00$ & $0.90 \pm 0.30$ & $0.90 \pm 0.30$ & $\infty$ & $0.00 \pm 0.00$ & $0.91 \pm 0.30$ & $\mathrm{~T}$ \\
\hline $16 \mathrm{~A}$ & $0.00 \pm 0.00$ & $0.82 \pm 0.26$ & $0.82 \pm 0.26$ & $\infty$ & $0.00 \pm 0.00$ & $0.83 \pm 0.26$ & $\mathrm{~T}$ \\
\hline 16B & $0.00 \pm 0.00$ & $0.68 \pm 0.17$ & $0.68 \pm 0.17$ & $\infty$ & $0.00 \pm 0.00$ & $0.69 \pm 0.17$ & $\mathrm{~T}$ \\
\hline$X$ & $0.52 \pm 0.10$ & $2.09 \pm 0.19$ & $2.62 \pm 0.27$ & $4.04 \pm 0.88$ & $0.18 \pm 0.03$ & $2.65 \pm 0.28$ & ST \\
\hline $\mathrm{Y}$ & $0.00 \pm 0.00$ & $0.76 \pm 0.15$ & $0.76 \pm 0.15$ & $\infty$ & $0.00 \pm 0.00$ & $0.77 \pm 0.15$ & $\mathrm{~T}$ \\
\hline
\end{tabular}

Note: p: Short arm length, q: Long arm length, pq: Total length of chromosome, AR: Arm ratio, CI: Centromeric index, RL\%: Relative length chromosome, M: Metacentric, SM: Submetacentric, ST: Subtelocentric, T: Telocentric. Asterisk symbol indicates marker chromosome 
Table 3. Chromosome measurement and morphology of juvenile male C. brachyotis collected from the mangrove forest

\begin{tabular}{|c|c|c|c|c|c|c|c|}
\hline Chromosome & $\mathbf{p}$ & $\mathbf{q}$ & $\mathbf{p q}$ & $\mathbf{A R}$ & CI & RL\% & $\begin{array}{c}\text { Chromosome } \\
\text { shape }\end{array}$ \\
\hline $1 \mathrm{~A}$ & 2.69 & 2.81 & 5.50 & 1.05 & 0.49 & 5.50 & $\vec{M}$ \\
\hline 1B & 2.72 & 2.72 & 5.44 & 1.00 & 0.50 & 5.44 & M \\
\hline $2 \mathrm{~A}$ & 2.43 & 2.74 & 5.17 & 1.13 & 0.47 & 5.17 & M \\
\hline $2 \mathrm{~B}$ & 2.31 & 2.63 & 4.94 & 1.14 & 0.47 & 4.94 & M \\
\hline $3 \mathrm{~A}$ & 1.99 & 2.55 & 4.55 & 1.28 & 0.44 & 4.55 & M \\
\hline $3 \mathrm{~B}$ & 1.45 & 2.40 & 3.85 & 1.65 & 0.38 & 3.85 & M \\
\hline $4 \mathrm{~A}$ & 1.60 & 2.07 & 3.68 & 1.29 & 0.43 & 3.68 & M \\
\hline $4 \mathrm{~B}$ & 1.63 & 1.91 & 3.54 & 1.17 & 0.46 & 3.54 & M \\
\hline $5 \mathrm{~A}$ & 1.47 & 2.03 & 3.50 & 1.38 & 0.42 & 3.50 & M \\
\hline $5 \mathrm{~B}$ & 1.63 & 1.79 & 3.43 & 1.10 & 0.48 & 3.43 & M \\
\hline $6 \mathrm{~A}$ & 1.25 & 1.96 & 3.21 & 1.57 & 0.39 & 3.21 & M \\
\hline $6 \mathrm{~B}$ & 1.25 & 1.95 & 3.21 & 1.56 & 0.39 & 3.21 & M \\
\hline $7 \mathrm{~A}$ & 1.02 & 1.28 & 2.30 & 1.25 & 0.44 & 2.30 & M \\
\hline $7 \mathrm{~B}$ & 0.83 & 0.97 & 1.81 & 1.17 & 0.46 & 1.81 & M \\
\hline $8 \mathrm{~A}$ & 0.62 & 0.94 & 1.56 & 1.53 & 0.40 & 1.56 & M \\
\hline $8 \mathrm{~B}$ & 0.60 & 0.68 & 1.28 & 1.12 & 0.47 & 1.28 & M \\
\hline $9 A^{*}$ & 1.40 & 3.05 & 4.45 & 2.18 & 0.31 & 4.45 & SM \\
\hline $9 B^{*}$ & 1.38 & 2.64 & 4.02 & 1.90 & 0.34 & 4.02 & SM \\
\hline $10 \mathrm{~A}$ & 1.20 & 2.19 & 3.40 & 1.82 & 0.35 & 3.40 & SM \\
\hline $10 \mathrm{~B}$ & 1.18 & 2.05 & 3.23 & 1.75 & 0.37 & 3.23 & SM \\
\hline $11 \mathrm{~A}$ & 1.10 & 2.02 & 3.12 & 1.84 & 0.35 & 3.12 & SM \\
\hline $11 \mathrm{~B}$ & 0.95 & 2.01 & 2.96 & 2.11 & 0.32 & 2.96 & SM \\
\hline $12 \mathrm{~A}$ & 0.78 & 2.01 & 2.79 & 2.56 & 0.28 & 2.79 & SM \\
\hline $12 \mathrm{~B}$ & 0.92 & 1.69 & 2.61 & 1.83 & 0.35 & 2.61 & SM \\
\hline $13 \mathrm{~A}$ & 0.38 & 1.90 & 2.28 & 4.99 & 0.17 & 2.28 & ST \\
\hline $13 \mathrm{~B}$ & 0.34 & 1.89 & 2.23 & 5.59 & 0.15 & 2.23 & ST \\
\hline $14 \mathrm{~A}$ & 0.00 & 1.63 & 1.63 & $\infty$ & 0.00 & 1.63 & $\mathrm{~T}$ \\
\hline 14B & 0.00 & 1.49 & 1.49 & $\infty$ & 0.00 & 1.49 & $\mathrm{~T}$ \\
\hline $15 \mathrm{~A}$ & 0.00 & 1.12 & 1.12 & $\infty$ & 0.00 & 1.12 & $\mathrm{~T}$ \\
\hline $15 B$ & 0.00 & 1.09 & 1.09 & $\infty$ & 0.00 & 1.09 & $\mathrm{~T}$ \\
\hline $16 \mathrm{~A}$ & 0.00 & 1.08 & 1.08 & $\infty$ & 0.00 & 1.08 & $\mathrm{~T}$ \\
\hline 16B & 0.00 & 0.90 & 0.90 & $\infty$ & 0.00 & 0.90 & $\mathrm{~T}$ \\
\hline$X$ & 0.50 & 3.22 & 3.72 & 6.47 & 0.13 & 3.72 & ST \\
\hline $\mathrm{Y}$ & 0.00 & 0.95 & 0.95 & $\infty$ & 0.00 & 0.95 & $\mathrm{~T}$ \\
\hline
\end{tabular}

Note: p: Short arm length, q: Long arm length, pq: Total length of chromosome, AR: Arm ratio, CI: Centromeric index, RL\%: Relative length chromosome, M: Metacentric, SM: Submetacentric, ST: Subtelocentric, T: Telocentric. Asterisk symbol indicates marker chromosome

\section{Discussion}

According to morphological studies, including the variation of forearm length, tail length, tibia length, and body weight, Corbet and Hill (1992) made 19 synonyms of C. brachyotis. Hereinafter, Mickleburgh et al. (1992) proposed nine subspecies names for this species, but Kitchener and Maharadatunkamsi (1991) regarded $C$. brachyotis into seven species, which $C$. $b$. javanicus distributed in Java, Madura, and Bali. Several studies were showed that two different lineages of $C$. brachyotis namely C. brachyotis Sunda and C. brachyotis Forest from Malaysia and Borneo have different morphology, especially in forearm length and body size (Abdullah 2003; Campbell et al. 2004).

The adults of $C$. brachyotis Sunda have forearm length more than $64 \mathrm{~mm}$ and inhabits open habitats such as fragmented areas, agriculture, and suburban, whereas $C$. brachyotis Forest has forearm length of less than $64 \mathrm{~mm}$ which inhabits closed area like a primary and secondary forest. Moreover, the body size of $C$. brachyotis Sunda was morphologically larger than $C$. brachyotis Forest and both were co-existed in the forest edge area (Abdullah 2003; Campbell et al. 2004). This study indicated otherwise. Adults C. brachyotis from closed habitats have higher forearm length and larger than $C$. brachyotis from open habitat, but it remains unclear from which lineage $C$. brachyotis in this study came. A further phylogenetic study is needed to know the lineages of $C$. brachyotis from different habitats in Yogyakarta.

The best metaphase slide of all samples in this study was not obtained because most chromosomes were condensed and were not spread properly. It is suspected due to Colcemid (Colchicine) injection and treatments with the hypotonic solution. Few crucial steps may affect the quality of chromosomal preparation results. Insufficient Colcemid incubation time was made fewer and longer metaphase spreads, then chromosomes will be overlapped. Whereas, the longer incubation times made thicker and shorter chromosomes (Howe et al. 2014). We already used two hours of incubation according to Baker et al. (1982) and we have also conducted a preliminary study in the laboratory. However, it seems not good enough for chromosomal preparation of this fruit bats species in the fieldwork. Colcemid treatments for a bit shorter will make chromosomes not so condensed for this study (Mollard R 2020, pers. com). 
Table 4. Chromosome measurement and morphology of adult female C. brachyotis collected from the urban area.

\begin{tabular}{|c|c|c|c|c|c|c|c|}
\hline Chromosome & $\mathbf{p}$ & $\mathbf{q}$ & pq & $\mathbf{A R}$ & CI & RL\% & $\begin{array}{c}\text { Chromosome } \\
\text { shape }\end{array}$ \\
\hline $1 \mathrm{~A}$ & 2.34 & 2.57 & 4.9 & 1.1 & 0.48 & 4.90 & $\mathrm{M}$ \\
\hline 1B & 2.08 & 2.74 & 4.83 & 1.32 & 0.43 & 4.83 & M \\
\hline $2 \mathrm{~A}$ & 2.07 & 2.45 & 4.51 & 1.18 & 0.46 & 4.51 & M \\
\hline $2 \mathrm{~B}$ & 1.95 & 2.5 & 4.45 & 1.28 & 0.44 & 4.45 & M \\
\hline $3 \mathrm{~A}$ & 1.91 & 2.33 & 4.24 & 1.21 & 0.45 & 4.24 & M \\
\hline $3 \mathrm{~B}$ & 1.91 & 2.31 & 4.21 & 1.21 & 0.45 & 4.21 & $\mathrm{M}$ \\
\hline $4 \mathrm{~A}$ & 1.76 & 1.88 & 3.64 & 1.07 & 0.48 & 3.64 & $\mathrm{M}$ \\
\hline $4 \mathrm{~B}$ & 1.62 & 1.90 & 3.53 & 1.17 & 0.46 & 3.53 & $\mathrm{M}$ \\
\hline $5 \mathrm{~A}$ & 1.63 & 1.88 & 3.51 & 1.15 & 0.46 & 3.51 & M \\
\hline $5 \mathrm{~B}$ & 1.54 & 1.84 & 3.38 & 1.2 & 0.46 & 3.38 & M \\
\hline $6 \mathrm{~A}$ & 1.38 & 1.82 & 3.2 & 1.32 & 0.43 & 3.20 & M \\
\hline $6 \mathrm{~B}$ & 1.26 & 1.62 & 2.88 & 1.29 & 0.44 & 2.88 & M \\
\hline $7 \mathrm{~A}$ & 1.13 & 1.34 & 2.47 & 1.19 & 0.46 & 2.47 & M \\
\hline $7 \mathrm{~B}$ & 1.00 & 1.22 & 2.22 & 1.22 & 0.45 & 2.22 & M \\
\hline $8 \mathrm{~A}$ & 0.84 & 0.89 & 1.73 & 1.07 & 0.49 & 1.73 & M \\
\hline $8 \mathrm{~B}$ & 0.76 & 0.87 & 1.62 & 1.14 & 0.47 & 1.62 & M \\
\hline $9 A^{*}$ & 1.04 & 2.62 & 3.66 & 2.52 & 0.28 & 3.66 & SM \\
\hline $9 B^{*}$ & 1.28 & 2.29 & 3.57 & 1.78 & 0.36 & 3.57 & SM \\
\hline $10 \mathrm{~A}$ & 1.05 & 2.13 & 3.19 & 2.03 & 0.33 & 3.19 & SM \\
\hline $10 \mathrm{~B}$ & 0.98 & 1.87 & 2.85 & 1.92 & 0.34 & 2.85 & SM \\
\hline $11 \mathrm{~A}$ & 0.67 & 3.19 & 3.86 & 4.78 & 0.17 & 3.86 & ST \\
\hline $11 \mathrm{~B}$ & 0.57 & 3.15 & 3.71 & 5.57 & 0.15 & 3.71 & ST \\
\hline $12 \mathrm{~A}$ & 0.78 & 2.79 & 3.57 & 3.58 & 0.22 & 3.57 & ST \\
\hline $12 \mathrm{~B}$ & 0.62 & 2.66 & 3.28 & 4.30 & 0.19 & 3.28 & ST \\
\hline $13 \mathrm{~A}$ & 0.32 & 1.43 & 1.75 & 4.40 & 0.18 & 1.75 & $\mathrm{ST}$ \\
\hline $13 \mathrm{~B}$ & 0.34 & 1.05 & 1.39 & 3.07 & 0.24 & 1.39 & $\mathrm{ST}$ \\
\hline $14 \mathrm{~A}$ & 0.00 & 1.59 & 1.59 & $\infty$ & 0.00 & 1.59 & $\mathrm{~T}$ \\
\hline $14 B$ & 0.00 & 1.46 & 1.46 & $\infty$ & 0.00 & 1.46 & $\mathrm{~T}$ \\
\hline $15 \mathrm{~A}$ & 0.00 & 1.40 & 1.40 & $\infty$ & 0.00 & 1.40 & $\mathrm{~T}$ \\
\hline $15 \mathrm{~B}$ & 0.00 & 1.39 & 1.39 & $\infty$ & 0.00 & 1.39 & $\mathrm{~T}$ \\
\hline $16 \mathrm{~A}$ & 0.00 & 1.24 & 1.24 & $\infty$ & 0.00 & 1.24 & $\mathrm{~T}$ \\
\hline $16 \mathrm{~B}$ & 0.00 & 1.09 & 1.09 & $\infty$ & 0.00 & 1.09 & $\mathrm{~T}$ \\
\hline $\mathrm{X}$ & 0.57 & 2.42 & 2.99 & 4.26 & 0.19 & 2.99 & $\mathrm{ST}$ \\
\hline $\mathrm{X}$ & 0.38 & 2.31 & 2.69 & 6.10 & 0.14 & 2.69 & ST \\
\hline
\end{tabular}

Note: p: Short arm length, q: Long arm length, pq: Total length of chromosome, AR: Arm ratio, CI: Centromeric index, RL\%: Relative length chromosome, M: Metacentric, SM: Submetacentric, ST: Subtelocentric, T: Telocentric. Asterisk symbol indicates marker chromosome

Molarity and treatment time of the hypotonic $\mathrm{KCl}$ solution was also important in the chromosome preparations. The hypotonic solution of $0.075 \mathrm{M}$ will make cell swelling enough (without lysing cells) for proper chromosomal spread (Howe et al. 2014). Hypotonic treatment for a bit longer (one or two minutes) in this study will make for better chromosome spreading (Mollard R 2020 , pers. com). The samples from a natural forest were not spread properly. Other factors that influence chromosome spreading were temperature and humidity. Those factors will affect the cell suspension drying time on the slide. Since the average temperature in the Tlogo Nirmolo was $21.8{ }^{\circ} \mathrm{C}$ and humidity was $93 \%$, it made the cell suspension relatively slow to air-drying. The best temperature and humidity for chromosome preparations were approximately $20-25^{\circ} \mathrm{C}$ and $50 \%$, respectively (Howe et al. 2014).

Ando et al. (1980) showed C. brachyotis karyotype from West Java having diploid number $2 n=34, F N a=58$ with 11 pairs of undistinguished metacentric, and submetacentric, two pair of medium subtelocentric and three pairs of minute acrocentric autosome. According to that results, both $C$. brachyotis from mangrove and urban areas in this study showed different karyotypes. The main differences were on subtelocentric numbers and classification of the acrocentric chromosomes. The acrocentric chromosomes were not classified by the software in this study. Acrocentric chromosome (synonyms to $t$ letter term) have a centromeric position in the terminal region ( $\mathrm{t}$ region with arm ratio=7.0) (Levan et al. 1964), while all chromosomes that classified as telocentric in this study have centromeric position in the terminal sensu stricto (arm ratio= $=\infty$ ), and symbolized with $\mathrm{T}$ letter. Furthermore, $\mathrm{X}$ and $\mathrm{Y}$ chromosomes are similar to the sex chromosomes of species from West Java and Malaysia that described by Yong et al. (1973) and Ando et al. (1980). Those sex chromosomes of C. brachyotis are characterized by medium $\mathrm{X}$ subtelocentric and very small $\mathrm{Y}$ acrocentric chromosomes. 
Karyotypes of $C$. brachyotis is having chromosome markers that are classified as medium metacentric in Malaysia and the Philippines, medium submetacentric in another Malaysia region, and also meta-submetacentric in West Java (Yong et al. 1973; Ando et al. 1980; Harada and Kobayashi 1980; Rickart et al. 1989). In this study, chromosome marker is classified as a medium submetacentric. Ando et al. (1980) also stated that chromosome marker position is considered as the fourth largest autosome in the karyotypes with relatives length of about $8.41 \pm 0.40$. This study indicates the same result with the smaller relative length. Otherwise, adult male bat from mangrove showed a different result. This condition was not common and still unclear since genus Cynopterus from Malaysia have uniformLy position of chromosome marker as the fourth-longest autosome with secondary constriction (Yong et al. 1973). However, a karyotype study of $C$. sphinx from Thailand showed the position of chromosome marker as the sixth (Harada et al. 1982). Therefore, It is suspected that the marker position of $C$. brachyotis was also varied.

Chromosome C. brachyotis from West Java has a higher relative length compared to this study. The relative length of West Java samples autosome ranged from $10.03 \pm 0.33$ to $0.99 \pm 0.22$ (Ando et al. 1980), whereas relative length in $C$. brachyotis Yogyakarta ranged from $6.78 \pm 0.59$ to $0.69 \pm 0.17$. Then, the relative length of sex chromosome $C$. brachyotis from West Java including $\mathrm{X}$ chromosome 5.58 \pm 0.18 and $\mathrm{Y}$ chromosome $1.58 \pm 0.58$ (Ando et al. 1980) were also higher than all samples from Yogyakarta.

Although chromosomes of bats in this study show different morphology and size but their fundamental number of autosomes still the same as in previous studies. C. brachyotis has a very stable diploid number and a fundamental number of autosome as other species of Cynopterus, such as C. horsfieldii and C. sphinx (Yong et al. 1973; Ando et al. 1980; Harada and Kobayashi 1980; Rickart et al. 1989). Diploid number and karyotypes of genus Cynopterus is though to be near from primitive karyotypes of family Pteropodidae. The primitive karyotype of Ptrepodidae has been proposed to have $2 n=36$ and $\mathrm{FN}=68$ (or close to it) with the domination of bi-armed autosomes, a pair of marker chromosomes, and bi-armed $\mathrm{X}$ chromosomes (SM or ST) (Ando et al. 1980; Hood et al. 1988). The diploid number trend of Pteropodidae was 34, 36, and 38 (Haiduk et al. 1980). Karyotypes of $C$. brachyotis are included in this trend.

Cynopterus belongs to the subfamily Cynopterinae, along with Ptenochirus, Megaerops, Dyacopterus, Balionycteris, Chironax, Thoopterus, Sphaerias, Aethalops, Penthetor, Latidens, Alionycteris, Otopteropus, and Haplonycteris genera (Bergmans 1997; Simmons and Cirranello 2020). Some karyotype studies in this subfamily are already conducted Southeast Asia. Diploid number of this subfamily members have wide range, reported as $2 \mathrm{n}=24 \quad$ (Balionycteris), $2 \mathrm{n}=26 \quad$ (Megaerops), $2 \mathrm{n}=28$ (Panthetor), 2n=34 (Cynopterus, Aethalops), $2 \mathrm{n}=36$ (Alionycteris), $2 \mathrm{n}=38 \quad$ (Thoopterus), and $2 \mathrm{n}=48$ (Otopteropus). Moreover, fundamental number autosomes were also different (Yong and Dhaliwal 1976; Harada and Kobayashi 1980; Harada et al. 1982; Hood et al. 1988; Rickart et al. 1999; Mubarok et al. 2018). It showed that this subfamily has very diverse karyotypes. The karyotype of Cynopterus is completely different from other pteropodids species, especially genus Pteropus because of pericentric inversion in metacentric chromosomes (SoteroCaio et al. 2017).

C. brachyotis is a very well-known and widespread species of the genus Cynopterus in different habitats. Based on this study result, this species is suspected to have different chromosome morphology (polymorphism). Chromosomal polymorphism event in bats has occurred in widespread species of vespertilionid bats Pipistrellus javanicus. That species showed different diploid numbers, ranged from 38 and 34 with the fundamental number of autosomes 48 and 46 respectively. Those differences are suggested because of Robertsonian translocation and also other chromosomal rearrangements (Rickart et al. 1999). On the other hand, this event in $C$. brachyotis and karyotypes data from other localities with many metaphase plates of this species is needed for further study.

In conclusion, lesser short-nosed fruit bat C.brachyotis that collected from three different habitats, i.e. urban area, natural forest, and mangrove forest in the special region Yogyakarta, Indonesia showed karyotype variation. The variation was observed in the chromosome shape which regarded the number of subtelocentric chromosomes and different relative lengths of chromosomes.

\section{ACKNOWLEDGEMENTS}

We indebted to Rekognisi Tugas Akhir funding number 2488/UN1.P.III/DIT-LIT/PT/2020 from Universitas Gadjah Mada. We also thank Beasiswa Lima Ribu Doktor (Mora) from the Ministry of Religious Affairs of the Republic of Indonesia for the first author doctoral study. All authors were contributed and wrote this paper.

\section{REFERENCES}

Abdullah MT. 2003. Biogeography and Variation of Cynopterus brachyotis in Southeast Asia. [Dissertation]. University of Queensland, Queensland.

Abramoff MD, Magalhaes PJ, Ram SJ. 2004. Image processing with image. J Biophotonics Int 11: 36-42.

Andersen K. 1912. Catalogue of the Chiroptera in the Collection of the British Museum. Megachiroptera 1. Taylor and Francis, London.

Ando K, Tagawa T, Uchida TA. 1980. A karyotypic study on four species of the Indonesian fruit-eating bats, belonging to Cynopterus, Eonycteris and Macroglossus (Chiroptera: Pteropidae). Caryologia 33: 41-53. DOI: 10.1080/00087114.1980.10796818.

Baker RJ, Haiduk MW, Robbins LW, Cadena A, Koop BF. 1982. Chromosomal studies of South American bats and their systematic implications. In: Mares MA, Genoways HH (eds) Mammalian Biology in South America. University of Pittsburg, Pennsylvania.

Bergmans W. 1997. Taxonomy and biogeography of African fruit bats (Mammalia, Megachiroptera). 5. the genera Lissonycteris Andersen, 1912, Myonycteris Matschie, 1899 and Megaloglossus Pagenstecher, 1885; General remarks and conclusions; Annex: key to all species. Beaufortia 47: 11-90. 
Burgin CJ, Wilson DE, Mittermeier RA, Rylands AB, Lacher TE, Sechrest W. 2020. Illustrated Checklist of The Mammals of The World. Lynx Ediciones, Spain.

Campbell P, Schneider CJ, Adnan AM, Zubaid A, Kunz TH. 2004. Phylogeny and phylogeography of old world fruit bats in the Cynopterus brachyotis complex. Mol Phylogenet Evol 33: 764-781. DOI: 10.1016/j.ympev.2004.06.019.

Corbet GB, Hill JE. 1992. The Mammals of the Indomalayan Region: A Systematic Review. Oxford University Press, Oxford.

Francis CM, Borisenko AV, Ivanova NV, Eger JL, Lim BK, GuillénServent A, Kruskop SV, Mackie I, Hebert PDN. 2010. The role of DNA Barcodes in understanding and conservation of mammal diversity in Southeast Asia. PLOS ONE 5: 1-12. DOI: 10.1371/journal.pone.0012575.

Haiduk MW, Robbins LW, Robbins RL, Schlitter DA. 1980. Karyotype studies of seven species of African megachiropterans (Mammalia: Pteropodidae). Ann Carnegie Mus 49: 181-191.

Harada M, Kobayashi T. 1980. Studies on the small mammal fauna of Sabah, East Malaysia II. Karyological analysis of some Sabahan mammals (Primates, Rodentia, Chiroptera). Contributions of the Biology Laboratory, Kyoto University 26: 83-95.

Harada M, Minezawa M, Takada S, Yenbutra S, Nunpakdee P, Ohtani S. 1982. Karyological analysis of 12 species of bats from Thailand. Caryologia 35: 269-278. DOI: 10.1080/00087114.1982.10796932.

Hood CS, Schlitter DA, Georgudaki JI, Baker RJ. 1988. Chromosome studies of bats (Mammalia: Chiroptera) from Thailand. Ann Carnegie Mus 57: 99-109.

Howe B, Umrigar A, Tsien F. 2014. Chromosome preparation from cultured cells. J Vis Exp 83: 1-5. DOI:10.3791/50203.

Huang JC, Ariyanti ES, Rustiati EL, Daaras K, Maryanto I, Maharadatunkamsi, Nusalawo M, Kingston T, Wiantoro S. 2016. The identification keys of bats in Sumatera: from the survey in Bukit Barisan Selatan. www.seabcru.org [Indonesian]

Kitchener DJ, Maharadatunkamsi. 1991. Description of a new species of Cynopterus (Chiroptera: Pteropodidae) from Nusa Tenggara, Indonesia. Rec West Aust Mus 15: 307-363.

Levan A, Fredga K, Sandberg AA. 1964. Nomenclature for centromeric position on chromosomes. Hereditas 52: 201-220.

Maryanto I. 1993. Activities of seed dispersing bats (Cynopterus brachyotis) in using ripe fruits at upper Cisadane watershed. Zoo Indonesia 17: 1-7. [Indonesian]

Maryanto I, Higashi S. 2011. Comparison of zoogeography among rats, fruit bats and insectivorous bats on Indonesian islands. Treubia 38: 33-52.

Maryati M, Kartono AP, Maryanto I. 2008. Pollinator Bat identification based on pollen as feed resources at Linggarjati District-Ciremai
National Park, West Java. Jurnal Biologi Indonesia 4: 335-47. [Indonesian]

Mickleburgh SP, Hutson AM, Racey PA. 1992. Old World Fruit Bats. An Action Plan for Their Conservation. IUCN, Gland.

Mubarok H, Perwitasari-Farajallah D, Maryanto I. 2018. Karyotypes of fruit bats (Chiroptera, Pteropodidae) and rats (Rodentia, Muridae) from Mt. Bawakaraeng, South Sulawesi, Indonesia. Borneo Science 39: $1-15$.

Rickart EA, Marcier JA, Heaney LR. 1999. Cytogeography of Philippine bats (Mammalia: Chiroptera). P Biol Soc Wash 112: 453-469.

Rickart EA, Heaney LR, Rosenfeld MJ. 1989. Chromosomes of ten species of Philippine fruit bats (Chiroptera: Pteropodidae). P Biol Soc Wash 102: 520-531.

Sakamoto Y, Zacaro AA. 2009. LEVAN an ImajeJ plugin for morphological cytogenetic analysis of mitotic and meiotic chromosomes. http://www.rsbweb.nih.gov/ij/.

Simmons NB. 2005. Order Chiroptera. In: Wilson DE, Reeder DM (eds) Mammal Species of The World: A Taxonomic and Geographic Reference. Johns Hopkins University Press, Baltimore.

Simmons NB, Cirranello AL. 2020. Bat species of the world: a taxonomic and geographic database. www.batnames.org

Sotero-Caio CG, Baker RJ, Volleth M. 2017. Chromosomal evolution in Chiroptera. Genes 8: 1-25.

Suripto BA, Zakky MK, Djatmiko T. 2001. Diversity of fruit bats species (Megachiroptera) and their natural feeds in Kokap, Kulon Progo, Yogyakarta. Berkala Ilmiah Biologi 2: 669-683. [Indonesian]

Suripto BA, Sumaryati, Budi C. 2006. The species of fruit bats and their impact on the coconut "Nira" production in Kokap, Kulon Progo. Jurnal Perlindungan Tanaman Indonesia 12: 13-24. [Indonesian]

Suyanto A. 2001. Bats in Indonesia. Puslitbang Biologi-LIPI, Bogor. [Indonesian]

Suyanto A, Yoneda M, Maryanto I, Maharadatunkamsi, Sugardjito J. 2002. Checklist of The Mammals of Indonesia. Scientific Name and Distribution Area Table in Indonesia Including CITES, IUCN and Indonesian Category for Conservation. LIPI-JICA-PHKA, Bogor.

Yong HS, Dhaliwal SS, Lim BL, Teh KL, Start AN. 1973. Uniformity of karyotypes of the fruit bats Cynopterus (Mammalia: Chiroptera, Pteropidae). Malaysian J of Science 2: 19-23.

Yong HS, Dhaliwal SS. 1976. Chromosomes of the fruit-bat subfamily Macroglossinae from peninsular Malaysia. Cytologia 41: 85-89.

Young HA, Sarath G, Tobias CM. 2012. Karyotype variation is indicative of subgenomic and ecotypic differentiation in switchgrass. BMC Plant Biol 12: 117. DOI: 10.1186/1471-2229-12-117.

Zein MS, Fitriana YS. 2015. DNA barcoding of fruit bats community in Indonesia. Jurnal Biologi Indonesia 11: 51-62. [Indonesian] 